\title{
Comparison of duplex PCR and phenotypic analysis in differentiating Candida dubliniensis from Candida albicans from oral samples
}

\author{
Asanga Sampath ${ }^{1}$, Manjula Weerasekera ${ }^{*}$, Ayomi Dilhari ${ }^{1}$, Chinthika Gunasekara ${ }^{1}$, Uditha Bulugahapitiya ${ }^{2}$, \\ Neluka Fernando ${ }^{1}$ and Lakshman Samaranayake ${ }^{3}$
}

\begin{abstract}
Candida dubliniensis shares a wide range of phenotypic characteristics with Candida albicans including a common trait called germ tube positivity. Hence, laboratory differentiation of these two species is cumbersome. Duplex PCR analyses for C. albicans and C. dubliniensis was performed directly on DNA extracted from a total of 122 germ tube positive isolates derived from 100 concentrated oral rinse samples from a random cohort of diabetics attending a clinic in Sri Lanka. These results were confirmed by DNA sequencing of internal transcribed spacer (ITS) region of rDNA of the yeasts. Performance efficacy of duplex PCR was then compared with phenotypic identification using a standard battery of phenotypic tests. Of the 122 germ tube positive isolates three were identified by duplex PCR as C. dubliniensis and the remainder as C. albicans. On the contrary, when the standard phenotypic tests, sugar assimilation and chlamydospore formation, were used to differentiate the two species 13 germ tube positive isolates were erroneously identified as C. dubliniensis. Duplex PCR was found to be rapid, sensitive and more specific than phenotypic identification methods in discriminating C. dubliniensis from C. albicans. This is also the first report on the oral carriage of $C$. dubliniensis in a Sri Lankan population.
\end{abstract}

Keywords: Candida albicans, Candida dubliniensis, Duplex PCR, Diabetes, Oral rinse

\section{Introduction}

Candida dubliniensis is an emerging medically relevant pathogenic yeast (Sullivan et al. 1995) associated with oral, vaginal, and systemic infections particularly in patients with human immunodeficiency virus infection and diabetes mellitus (Krcmery and Barnes 2002; Sullivan et al. 1995). C. dubliniensis and C. albicans are known to share many morphological and physiological characteristics such as germ tube positivity, production of chlamydospores and similar biochemical profiles leading to common misidentification of these two species (Sullivan and Coleman 1998; Sullivan et al. 1995). Also, variations

\footnotetext{
*Correspondence: mmweera@yahoo.com; mmweera@sjp.ac.lk ${ }^{1}$ Department of Microbiology, Faculty of Medical Sciences, University of Sri Jayewardenepura, Gangodawila, Nugegoda, Sri Lanka Full list of author information is available at the end of the article
}

in growth conditions including incubation temperature, repeated sub-culture, and storage may impede their accurate identification (Pasligh et al. 2008). Furthermore, both these species are now known to be increasingly resistant to commonly used azole antifungal agents. Given their importance in common oral and systemic infections, there is a critical need to accurately and rapidly identify these pathogens for better patient management.

Several phenotypic and genotypic tests have been developed, validated and applied to differentiate C. albicans from C. dubliniensis. Commercially available yeast identification systems (e.g. Vitek 2 ID-YST, API 20C and ID32C) based on utilization of various compounds are the most popular current methods used in clinical laboratories for this purpose, although they are relatively expensive and requires up to $2-3$ days incubation 
to obtain results (Pincus et al. 1999). On the other hand, polymerase chain reaction PCR (Ellepola et al. 2003) and duplex PCR (Ahmad et al. 2012) have been recently used as genotypic tests for rapid identification and differentiation of the two species.

Here we report a comparison of well described phenotypic methods with the duplex PCR assay (Ahmad et al. 2012) using primers derived from unique ribosomal deoxyribonucleic acid (rDNA) sequences for rapid detection and differentiation of C. albicans and C. dubliniensis. The was assays were performed using yeast isolates derived from oral rinse samples from a cohort of diabetic patients attending a clinical facility in Colombo, Sri Lanka.

\section{Materials and methods}

\section{Patients and yeast isolates}

Concentrated oral rinse samples were randomly collected from 250 patients with type 2 diabetes mellitus, attending the Diabetes and Endocrinology Unit at a Tertiary Care Hospital in Sri Lanka, as per the method of Samaranayake et al. (1986). In brief, the patients were given $10 \mathrm{ml}$ of sterile phosphate-buffered saline and advised to rinse their mouths for $60 \mathrm{~s}$ and expectorate into the provided container. Each specimen was immediately taken to the laboratory, vortex mixed and centrifuged at $(6000 \mathrm{rpm})$ $3300 \times g$ for $10 \mathrm{~min}$. The pellet obtained from the rinse specimen was re-suspended in $1 \mathrm{ml}$ of sterile phosphate buffered saline. Hundred microliter of the re-suspended specimens was cultured on Sabouraud Dextrose Agar and incubated at $37{ }^{\circ} \mathrm{C}$ for $72 \mathrm{~h}$ and the resultant growth evaluated for yeast growth.

Subsequently, one to two colony phenotypes (colony forming units, CFUs) that resemble different yeast species were randomly selected from each sample. These CFUs were subcultured for $18 \mathrm{~h}$ to obtain a pure growth and harvested, and phenotypic and genotypic analysis performed to differentiate the two species, as described below.

For standardization purposes two reference isolates: C. albicans American Type Culture Collection (ATCC) 10231, C. dubliniensis ATCC MYA 580 and C. dubliniensis ATCC MYA 577 obtained from the Department of Oral Biology, University of Hong Kong, China, were used.

\section{Phenotypic analysis}

Of the 250 patient samples collected, 204 (81.6\%) yielded a yeast growth on culture. The culture plates were then examined by a single examiner and up to two different phenotypes form each sample were selected for the germ tube test (Isibor et al. 2005). The final analysis was then performed as follows: first, the rinse pellets derived from randomly selected 100 samples that yielded germ tube positive yeasts were subjected to duplex PCR. Then, 122 germ tube positive yeast isolates from the aforementioned (100) samples were subjected to both phenotypic analysis and genotypic analysis (i.e. duplex PCR) as described below. The phenotypic tests used for speciation of 122 isolates were chlamydospore production test (Kim et al. 2002), growth at $42{ }^{\circ} \mathrm{C}$ (Sullivan et al. 1995), and assimilation of xylose and trehalose (Pincus et al. 1999).

\section{DNA extraction, duplex PCR assay and sequence identification}

DNA extraction was performed using the conventional bead beater method (Sambrook and Rusell 2006) with modifications. A loopful of isolated colonies or pellet of the concentrated oral rinse sample was suspended in $100 \mu \mathrm{l}$ STES buffer [200 mM Tris-HCl (pH 7.6), $100 \mathrm{mM}$ EDTA (Ethylenediaminetetraacetic acid), $0.1 \%$ SDS (sodium dodecyl sulfate)] and $40 \mu \mathrm{l}$ of TE (Tris-EDTA) buffer [10 mM Tris-HCl (pH 8), 1 mM EDTA], $120 \mu \mathrm{l}$ phenol: chloroform mixture $(1: 1 \mathrm{~V} / \mathrm{V})$ and $0.3 \mathrm{~g}$ sterile zirconium beads ( $0.1 \mathrm{~mm}$ diameter; Bio Spec-Products) were added. The samples were homogenized using a mini bead beater (model 3110BX; Bio Spec Products) at $480 \mathrm{rpm}$ for $5 \mathrm{~min}$. The upper aqueous phase $(100 \mu \mathrm{l})$ was transferred to a sterile micro centrifuge tube, and DNA was precipitated in the presence of $220 \mu \mathrm{l}$ cold ethanol $(100 \%)$ and $10 \mu \mathrm{l}$ of $3 \mathrm{M}$ sodium acetate at $-20{ }^{\circ} \mathrm{C}$ for $18 \mathrm{~h}$. The solution was centrifuged at $(13,000 \mathrm{rpm})$ $15,493 \times g$ for $12 \mathrm{~min}$ and the DNA pellet was air dried and dissolved in $30 \mu \mathrm{l}$ TE buffer. Extracted DNA samples were stored at $-20^{\circ} \mathrm{C}$ until used.

Extracted DNA were subjected to the quantification using NANO drop 2000/200C spectrophotometer (Thermo Fisher Scientific, USA). Extracted DNA from oral rinse specimens and germ tube positive isolates were subjected to duplex PCR to differentiate $C$. albicans from C. dubliniensis.

Species-specific identity of C. albicans and C. dubliniensis strains were performed by duplex PCR using primers targeting sequences in ITS-1 (internal transcribed spacer-1) and ITS-2 regions of rDNA (Ahmad et al. 2012), respectively. Species-specificity of primer pairs for C. albicans and C. dubliniensis are shown in Table 1.

Duplex PCR amplification was carried out in a final volume of $50 \mu \mathrm{l}$ with $2 \mu \mathrm{l}$ template DNA, $1 \times$ green Go Taq Flexi buffer (pH 8.5), $3 \mathrm{mM} \mathrm{MgCl}, 0.2 \mu \mathrm{M}$ of each primer $(C A L F+C A L R+C D U F+C D U R)($ Table 1$)$, $0.2 \mathrm{mM}$ Deoxy Nucleotide Triphosphate (dNTP) mix and 1.25 unit of Go Taq DNA polymerase (Promega, USA). PCR amplification was done using GeneAmp PCR systems 9700 (Applied Bio systems, USA). The PCR reaction was initiated at $95{ }^{\circ} \mathrm{C}$ for $5 \mathrm{~min}$ followed by 30 cycles of $95{ }^{\circ} \mathrm{C}$ for $1 \mathrm{~min}, 55^{\circ} \mathrm{C}$ for $30 \mathrm{~s}$ and $72{ }^{\circ} \mathrm{C}$ for $1 \mathrm{~min}$ and a 
Table 1 The primer sequences used for amplification of C. albicans and C. dubliniensis for duplex PCR

\begin{tabular}{lllll}
\hline Target gene & Target species & Primer & Primer sequence $\left(\mathbf{5}^{\prime}->\mathbf{3}^{\prime}\right)$ & Amplicon size (bp) \\
\hline ITS 2 & C. albicans & CALF & TGG TAA GGC GGG ATC GCTT & 100 \\
& & CALR & GGT CAA AGT TTG AAG ATA TAC & \\
ITS 1 & C. dubliniensis & CDLF & AAA CTT GTC ACG AGA TTA TTT TT & 325 \\
ITS 2 & & AAA GTTTGA AGA ATA AAATGG C & \\
\hline
\end{tabular}

final elongation at $72{ }^{\circ} \mathrm{C}$ for 10 min with final hold at $4{ }^{\circ} \mathrm{C}$. All PCR experiments included a negative (no template) control and a positive control. Resulting PCR products were separated by electrophoresis using $1 \times$ TAE (Tris base, acetic acid and EDTA) (40 mM Tris $\mathrm{HCl}(\mathrm{pH} 8)$, $20 \mathrm{mM}$ acetic acid, $1 \mathrm{mM}$ EDTA) on a $3 \%(\mathrm{w} / \mathrm{v})$ agarose gel, stained with ethidium bromide and viewed by UV (Ultra Violet) trans-illuminator (Vilber Lourmat, QUANTUM ST4).

The PCR products derived from isolates which were identified as C. dubliniensis by duplex PCR were purified using a pureLink ${ }^{\mathrm{TM}}$ Quick Gel Extraction and PCR Purification Combo Kit (Invitrogen, Thermo Fisher Scientific, and USA) and were subjected to sequencing using PCR primers in both directions. The DNA sequences obtained were aligned using BioEdit and subjected to National Center for Biotechnology Information (NCBI) Blast to identify the species.

\section{Results}

\section{Clinical data}

Of the 250 oral rinse samples collected, 204 (81.6\%) yielded a yeast growth on culture. When the germ tube test was conducted 167 (81.8\%) of the latter cohort were carrying germ tube positive yeasts, and the remainder germ tube negative yeasts. We then randomly selected 100 patient samples which were germ tube positive, and this cohort was carrying 122 germ tube positive yeast isolates that were selected for the final analysis (some patients carried two, germ tube positive phenotypes). The rinse pellets derived from the foregoing samples were also subjected to duplex PCR in parallel. It is also noteworthy, that out of the selected 100 culture positive patients majority 67 per cent $(67 / 100)$ had yeast colony counts over 600 colony-forming units (CFU) per ml of the concentrated oral rinse sample.

\section{Duplex PCR of the concentrated oral rinse samples}

When the extracted DNA from pellets of 100 concentrated rinse samples were directly subjected to duplex PCR three samples were positive for $C$. dubliniensis and the remainder were positive for $C$. albicans only. Interestingly, of the three $C$. dubliniensis positive patients one patient yielded both $C$. albicans and C. dubliniensis and two patients yielded only C. dubliniensis.

On duplex PCR, C. dubliniensis yielded a band around 325 bp positions while $C$. albicans gave a band at $100 \mathrm{bp}$ position (Fig. 1).

\section{Duplex PCR of the germ tube positive isolates}

The colonial yield from the 100 randomly selected patient samples were phenotypically evaluated for germ tube positivity a total of 122 germ tube positive strains were identified, and these were presumptively identified as either $C$. albicans or $C$. dubliniensis. When these were subjected to duplex PCR, three were identified as C. $d u b$ liniensis and the remainder as C. albicans.

Thus, the duplex PCR results from the pellet analysis of 100 patients or from analyzing the 122 germ tube positive yeasts derived from the same cohort were identical with three results that were positive.

\section{Phenotypic identification}

Of the 122 germ tube positive isolates those were subjected to sugar assimilation tests using xylose and trehalose, to differentiate C. albicans from C. dubliniensis, xylose was assimilated by 108 isolates, trehalose by 114 , and xylose and trehalose by 106 isolates. On this basis 16 isolates could be characterized as $C$. dubliniensis. However, when the identity of these 16 isolates were scrutinized by duplex PCR only three isolates were genotypically confirmed as C. dubliniensis.

On further analyzing the phenotype, of 122 isolates, 114 grew well at $42{ }^{\circ} \mathrm{C}$ and were identified as C. albicans and eight colonies which did not yield any growth at $42{ }^{\circ} \mathrm{C}$ and were presumptively identified as $C$. dubliniensis. However, when they were subjected to duplex PCR only one out of the eight isolates was definitively identified as $C$. dubliniensis. The test sensitivity and specificity of each phenotypic identification method was found to vary (Table 2 ).

\section{Comparison of duplex PCR and phenotypic identification}

Comparing duplex PCR results, considered as the gold standard, with the phenotypic methods, the latter had poor sensitivity, of $33.3 \%$, and relatively high specificity, 




Fig. 1 Duplex PCR results on the 2\% Agarose gel. L, 100 bp ladder; N, negative control; lane no 1-4, 6 and 8, C. albicans; lanes 7 and 9, C. dubliniensis; lane 5, positive for both C. albicans and C. dubliniensis

Table 2 The sensitivity and the specificity of the phenotypic methods used for differentiating C. albicans and C. dubliniensis (using duplex PCR identification as the gold standard)

\begin{tabular}{lllcc}
\hline Cultivation method & C. dubliniensis_sensitivity (\%) & C. albicans_specificity (\%) & PPV (\%) & NPV (\%) \\
\hline Chlamydospore formation & 33.3 & 97.48 & 25 & 98.31 \\
Growth at $42^{\circ} \mathrm{C}$ & 33.3 & 94.12 & 12.5 & 98.25 \\
Assimilation of xylose & 33.3 & 89.08 & 7.14 & 98.15 \\
Assimilation of trehalose & 33.3 & 94.12 & 12.5 & 98.25 \\
\hline
\end{tabular}

PPV positive predictive value, NPV negative predictive value

and a poor positive predictive value and high negative predicative values (Table 2).

\section{Discussion}

Oral infections with Candida species are on the rise. This is mainly due to the burgeoning immune compromised populations worldwide, such as those with human immunodeficiency virus (HIV) disease, and those on immuneand cytotoxic therapy. Additionally, the pre diabetics and diabetics are well known to have a high prevalence of oral Candida (Samaranayake 2011). Hence a rapid, reliable and an inexpensive method for identification of Candida, particularly C. albicans, the most virulent Candida species, from the less virulent surrogate, $C$. dubliniensis is clinically advantageous.

The duplex PCR assay described in this study enabled the accurate differentiation and identification of $C . d u b$ liniensis from $C$. albicans strains form oral rinses specimens. In total, the whole procedure could be completed in $4 \mathrm{~h}$ as opposed to up to 3 days required for the traditional, phenotypic tests. Further it requires minimal quantity of genomic DNA. Although this is not the first study to use duplex PCR to identify and distinguish $C$. dubliniensis and $C$. albicans, but it is the first to analyses oral samples directly through rinse pellet analysis, and samples from a Sri Lankan diabetic population using duplex PCR. 
Ahmad et al. reported duplex PCR application as an accurate tool for identification and differentiation of $C$. albicans and $C$. dubliniensis, with a very high index of sensitivity and specificity (Ahmad et al. 2012). In agreement, with the flatter findings we too noted that duplex PCR amplification with CALF + CALR + CDUF + CDUR primers yielded species-specific single amplicons of $\sim 100$ and $\sim 325$ bp of C. albicans and C. dubliniensis, respectively, that provided a high degree of discrimination. Further, duplex PCR was found to be a rapid, sensitive and simple technique, which could be directly applied to oral rinse specimens as well as clinical isolates derived from these rinses. It may be possible to extend the application to the other clinical specimens such as blood, saliva and fecal specimens, especially in septicemic states with important clinical impact and further studies are warranted for this purpose.

In the current study, the results of duplex PCR assay were not fully in agreement with species-specific phenotypic identification of $C$. dubliniensis and C. albicans. For instance, the growth at $42{ }^{\circ} \mathrm{C}$ poorly differentiated the two species. Others too have previously reported that growth at a higher temperature as an unreliable criterion for this purpose (Pasligh et al. 2010). Similarly, the assimilation data for trehalose and xylose also were not reliable and confirmed the findings of Tintelnot et al. who concluded that the observed pattern for the assimilation of xylose is not discriminating enough to differentiate the two species (Tintelnot et al. 2000). Finally, the corn meal and tween 80 agar tests to evaluate the degree of chlamydospore formation was also unreliable as one-third of both species produced these appendages (Table 2) This is consistent with the observations of previous workers who noted that both $C$. dubliniensis and $C$. albicans produce chlamydospores (Sancak et al. 2005; Sullivan and Coleman 1998; Sullivan et al. 1995) and querying its validity as a differential phenotypic trait.

In clinical terms, we noted that $67 \%$ of our Sri Lankan diabetic population yielded oral Candida concentrations greater than $600 \mathrm{CFU}$ per $\mathrm{ml}$ as evaluated by concentrated oral rinse culture indicating a rather heavy carriage of oral yeasts, and the consequent probability of overt yeast infection. Candida cell counts of $>600 \mathrm{CFU}$ per $\mathrm{ml}$ in oral rinse samples is considered as indicative of oral Candida infection according to previous workers (Samaranayake and MacFarlane 1990).

Others have reported oral carriage of Candida in patients with type 2 diabetes mellitus ranging from 13 to $64 \%$ (Fernandez et al. 2013). Such wide variation in oral Candidal carriage is a reflection of many confounding factors including the diabetes status and more crucially, the sampling method. It is well known that oral rinses samples yield a higher CFU count than the swab sampling method. The latter method excludes various oral niches preferred by Candida such as the posterior vestibular sulci (Samaranayake and MacFarlane 1990).

One interesting observation of the study was a single patient with dual species oral carriage of both C. albicans and C. dubliniensis. Revelation of such dual species carriage of phenotypically similar yeast isolates would be extremely difficult, if not time consuming and labor intensive, by conventional cultural techniques. The duplex PCR would therefore be useful to uncover the little described phenomenon of the multi species oral yeast carriage. For instance, Samaranayake et al. (1987) in a similar study revealed, up to $15 \%$ of oral rinse specimens in a British dental hospital yielded more than one yeast species, and hence duplex as well as multiplex PCR technology should be used by future workers to shed further light on this phenomenon (Samaranayake et al. 1987).

Finally, to our knowledge, this is first report of $C$. dubliniensis isolation and oral carriage in a Sri Lankan cohort. The 3\% prevalence of carriage reported here is similar to that of $2 \%$ oral and gastro intestinal prevalence reported by Odds et al. (1998) in 2589 yeasts in a stock collection from Europe, implying that $C$. dubliniensis is an opportunist pathogen of low consequence with similar worldwide prevalence profiles (Odds et al. 1998). Further clinical epidemiological data, particularly from the Asian region, are needed to confirm our preliminary findings.

\begin{abstract}
Abbreviations
PCR: polymerase chain reaction; DNA: deoxyribonucleic acid; ITS: internal transcribed spacer; rDNA: ribosomal deoxyribonucleic acid; ATCC: American Type Culture Collection; $\mathrm{HCl}$ : hydrochloric acid; EDTA: ethylenediaminetetraacetic acid; $\mathrm{MgCl}_{2}$ : magnesium chloride; SDS: sodium dodecyl sulfate; TE: tris-EDTA buffer solution; dNTP: deoxy nucleoside tri-phosphate; UV: ultra violet; TAE: tris base, acetic acid and EDTA; NCBI: National Center for Biotechnology Information; CFU: colony-forming units; HIV: human immunodeficiency virus.
\end{abstract}

\section{Authors' contributions}

MW, CG, and NF Conceived and designed the experiments. UB selected and recruited the patient for the study. AS and AD performed the experiments and analyzed the data. LS advised for the overall study. All engaged for writing of the manuscript. All authors read and approved the final manuscript.

\section{Author information}

AS obtained his B.Sc. in Molecular biology and biochemistry from Faculty of science, University of colombo and currently he is reading for his Ph.D. at University of Sri Jayewardenepura, Sri Lanka.

AD obtained her B.Sc. in Medical Laboratory Science from Faculty of Medical Sciences, University of Sri Jayewardenepura and currently she is reading for her Ph.D. at University of Sri Jayewardenepura, Sri Lanka.

MW is a senior lecturer attached to the Department of Microbiology, Faculty of Medical Sciences, University of Sri Jayewardenepura, Sri Lanka. She obtained her Ph.D. in molecular medicine in 2011 from the University of Otago, New Zealand. Her current research interests are antimicrobial resistance, bio-films studies and pathogenesis of medically important microorganisms

CG is a senior lecturer attached to the Department of Microbiology, Faculty of Medical Sciences, University of Sri Jayewardenepura, Sri Lanka.

NF is a professor attached to the Department of Microbiology, Faculty of Medical Sciences, University of Sri Jayewardenepura, Sri Lanka. 
UB is a Consultant Endocrinologist at Diabetes and Endocrinology Unit, Colombo South Teaching Hospital, Kalubowila, Sri Lanka.

$L S$ is a senior professor. His current research interests are in oral microbiomics (particularly biofilm biology), infection control, and dental education.

\section{Author details}

${ }^{1}$ Department of Microbiology, Faculty of Medical Sciences, University of Sri Jayewardenepura, Gangodawila, Nugegoda, Sri Lanka. ${ }^{2}$ Diabetes and Endocrinology Unit, Colombo South Teaching Hospital, Kalubowila, Dehiwala, Sri Lanka. ${ }^{3}$ Health Science Center, Kuwait University, Kuwait City, Kuwait.

\section{Acknowledgements}

We thank all patients, staff of endocrinology clinic at Colombo South Teaching Hospital, Sri Lanka and the members of the Department of Microbiology, Faculty of Medical Sciences and University of Sri Jayewardenepura, Sri Lanka for their assistance in the specimen collection. We would also like to thank Ms. Joyce Yau of Department of Oral Biology, University of Hong Kong for donating C. dubliniensis ATCC strains.

\section{Competing interests}

The authors declare that they have no competing interests.

\section{Availability of data and materials}

The data supporting the findings of this study are included in the main manuscript file and in the additional files.

\section{Consent to publish}

Consent to publish has been obtained from the participant to report individual patient data.

\section{Ethical approval}

Accepted principles of ethical and professional conduct. Ethical approval for this study was obtained from the Ethical Review Committee of University of Sri Jayewardenepura, Sri Lanka (Ref. No: 764/13) as well as the patients'approval was obtained prior to the sample collection.

\section{Funding}

This work was supported in part by grants from University of Sri Jayewardenepura, Sri Lanka (Grant No. ASP/06/RE/MED/2014/08) and by Medical Research Institute Sri Lanka. (Grant No: 2013/33).

\section{Publisher's Note}

Springer Nature remains neutral with regard to jurisdictional claims in published maps and institutional affiliations.

Received: 28 April 2017 Accepted: 16 June 2017

Published online: 27 June 2017

\section{References}

Ahmad S, Khan Z, Asadzadeh M, Theyyathel A, Chandy R (2012) Performance comparison of phenotypic and molecular methods for detection and differentiation of Candida albicans and Candida dubliniensis. BMC Infect Dis 12(1):230

Ellepola A, Hurst S, Elie C, Morrison C (2003) Rapid and unequivocal differentiation of Candida dubliniensis from other Candida species using species-specific DNA probes: comparison with phenotypic identification methods. Mol Oral Microbiol 18(6):379-388
Fernandez MRF, Jaimes-Aveldañez A, Hernandez-Perez F (2013) Oral Candida spp. carriers: its prevalence in patients with type 2 diabetes mellitus. An Bras Dermatol 88:222-225

Isibor J, Eghubare A, Omoregie R (2005) Germ tube formation in Candida albicans. Shiraz E-Med J 6(1 and 2):21-25

Kim D, Shin W, Lee K, Kim K, Park JY, Koh C (2002) Rapid differentiation of Candida albicans from other Candida species using its unique germ tube formation at $39^{\circ} \mathrm{C}$. Yeast 19:957-962

Krcmery V, Barnes A (2002) Non-albicans Candida spp. causing fungaemia: pathogenicity and antifungal resistance. J Hosp Infect 50(4):243-260

Odds FC, Van Nuffel L, Dams G (1998) Prevalence of Candida dubliniensis isolates in a yeast stock collection. J Clin Microbiol 36(10):2869-2873

Pasligh J, Radecke C, Fleischhacker M, Ruhnke M (2008) Comparison of phenotypic methods for the identification of Candida dubliniensis. J Microbiol Immunol Infect 43(2):147-154

Pasligh J, Radecke C, Fleischhacker M, Ruhnke M (2010) Comparison of phenotypic methods for the identification of Candida dubliniensis. J Microbiol Immunol Infect 43(2):147-154

Pincus D, Coleman D, Pruitt W, Padhye A, Salkin I, Geimer M, Bassel A, Sullivan D, Clarke M, Hearn V (1999) Rapid identification of Candida dubliniensis with commercial yeast identification systems. J Clin Microbiol 37(11):3533-3539

Samaranayake L (2011) Essential microbiology for dentistry. Elsevier Health Sciences, Amsterdam

Samaranayake LP, MacFarlane TW (1990) Oral candidosis. Wright Publishing Company, California

Samaranayake L, MacFarlane T, Lamey PJ, Ferguson M (1986) A comparison of oral rinse and imprint sampling techniques for the detection of yeast, coliform and Staphylococcus aureus carriage in the oral cavity. J Oral Pathol Med 15(7):386-388

Samaranayake L, MacFarlane T, Williamson M (1987) Comparison of Sabouraud dextrose and Pagano-Levin agar media for detection and isolation of yeasts from oral samples. J Clin Microbiol 25(1):162-164

Sambrook J, Rusell DW (2006) The condensed protocols, from molecular cloning: a laboratory manual. Cold Spring Harbor Laboratory Press, New York

Sancak B, Colakoglu S, Acikgoz ZC, Arikan S (2005) Incubation at room temperature may be an independent factor that induces chlamydospore production in Candida dubliniensis. Diagn Microbiol Infect Dis 52(4):305-309

Sullivan D, Coleman D (1998) Candida dubliniensis: characteristics and identification. J Clin Microbiol 36(2):329-334

Sullivan DJ, Westerneng TJ, Haynes KA, Bennett DE, Coleman DC (1995) Candida dubliniensis sp. nov:: phenotypic and molecular characterization of a novel species associated with oral candidosis in HIV-infected individuals. Microbiology 141(7):1507-1521

Tintelnot K, Haase G, Seibold M, Bergmann F, Staemmler M, Franz T, Naumann D (2000) Evaluation of phenotypic markers for selection and identification of Candida dubliniensis. J Clin Microbiol 38(4):1599-1608

\section{Submit your manuscript to a SpringerOpen ${ }^{\circ}$ journal and benefit from:}

- Convenient online submission

- Rigorous peer review

- Open access: articles freely available online

- High visibility within the field

- Retaining the copyright to your article

Submit your next manuscript at $\boldsymbol{\nabla}$ springeropen.com 